\title{
VARIABILIDADE DA PRECIPITAÇÃO EM TEMPO E ESPAÇO ASSOCIADA À ZONA DE CONVERGÊNCIA INTERTROPICAL
}

\author{
MAURÍCIO DO NASCIMENTO MOURA E MARIA ISABEL VITORINO
}

\author{
Universidade Federal do Pará (UFPA), Belém, PA, BRASIL \\ mauriciomoura90@bol.com.br, vitorino@ufpa.br
}

Recebido Abril de 2011 - Aceito Março de 2012

\begin{abstract}
RESUMO
Este estudo visa apresentar uma análise atmosférica da variabilidade espacial e temporal da Zona de Convergência Intertropical (ZCIT) nas cidades de Belém, Jakarta e Nairóbi, que estão localizadas sobre os continentes da América do Sul, Ásia e África, respectivamente. Para isso, foram utilizados dados diários de precipitação observada e radiação de onda longa para o período de 1999 a 2008, e aplicadas as técnicas matemáticas e estatísticas, como a média aritmética e a transformada em ondeletas Morlet. Em geral, os resultados indicam que do ponto de vista espacial, a precipitação mensal varia consideravelmente, pois as três cidades estudadas localizam-se em diferentes continentes da faixa tropical. Isto ocorre principalmente, durante os meses de Janeiro a Maio, período de maior atuação da ZCIT no hemisfério sul. As variações atmosféricas observadas, a partir dos escalogramas de fase, - de ondeleta indicam que as escalas interdecadal, anual, interanual e intrassazonal são moduladoras da precipitação. Tais escalas podem ser representadas pelos mecanismos oceano-atmosfera dos fenômenos El Niño Oscilação Sul e da oscilação intrassazonal de Madden e Julian. A contribuição destes fenômenos na distribuição da chuva nessas regiões é evidente durante o período estudado, sendo que Nairóbi, apesar de estar localizada em latitude semelhante à de Belém, apresenta pouca evidência do ciclo anual e forte na escala interdecadal. No caso de Belém e de Jakarta as oscilações de múltiescala de precipitação concentram-se nas escalas dos mecanismos moduladores da chuva associados com o ciclo anual e intrassazonal, durante todo o período.
\end{abstract}

Palavras-chave: Climatologia, ITCZ, Precipitação, Tropical.

\begin{abstract}
VARIABILITY OF RAINFALL IN TIME AND SPACE ASSOCIATED WITH INTERTROPICAL CONVERGENCE ZONE

This study aims to present an atmospheric analysis of spatial and temporal variability of the Intertropical Convergence Zone (ITCZ) in Belem, Jakarta and Nairobi, which are located on the continents of South America, Asia and Africa, respectively. For this, daily precipitation and observed long wave radiation data for the period 1999 to 2008 were used, and mathematical and statistical techniques, such as the arithmetic mean and the Morlet wavelet transform were applied. In general, the results indicate that spatially, the month rainfall varies considerably, since that the three studied cities are located far apart in different continents in the tropical zone. This occurs mainly during the months from January to May, period of greatest activity of the ITCZ in the southern hemisphere. The atmospheric variations observed from the phase wavelet scalograms indicate that the interdecadal scales, annual, interannual and intraseasonal scales are rainfall modulators. Such scales can be represented by the ocean-atmosphere phenomenon mechanisms of El Niño Southern Oscillation and intraseasonal oscillation of Madden and Julian. The contribution of these phenomena to the rainfall distribution over these regions is evident during the study period, and Nairobi, in spite of being located at latitude similar to that of Belem, shows low evidence of the annual cycle and high at interdecadal scale. In the case of Belem and Jakarta oscillations at multiscale rainfall concentrated in the mechanisms scales that modulate the rain associated with the annual cycle and intraseasonal cycle during the period.
\end{abstract}

Keywords: Climatology, ITCZ, Precipitation, Tropics. 


\section{INTRODUÇÃO}

Na região tropical, a variação tempo-espaço das variáveis meteorológicas, em especial a precipitação, está relacionada à atuação de fenômenos meteorológicos de diferentes escalas de tempo, que modulados por mecanismos oceano-atmosfera de escala interanual, sazonal e intrassazonal determinam a quantidade pluviométrica de determinadas regiões equatoriais. Neste sentido, os principais mecanismos tropicais de circulação oceano-atmosfera são: o El Niño Oscilação Sul, o Dipolo do Atlântico e a Oscilação de Madden-Julian que atuantes em suas diferentes fases favorecem ou desfavorecem a atividade convectiva em áreas tropicais (Madden e Julian, 1994; Moura e Shukla, 1981; Rasmusson e Arkin, 1985; Souza e Ambrizzi, 2006; Vitorino, 2003; 2006; Weickmann et al., 1985).

Dentre os fenômenos meteorológicos de grande escala, destaca-se a Zona de Convergência Intertropical (ZCIT), que é uma banda zonal associada à atividade convectiva que oscila entre $5^{\circ} \mathrm{S}$ e $10^{\circ} \mathrm{N}$, aproximadamente (Uvo, 1989; Waliser e Gautier, 1993). Este fenômeno caracteriza-se por uma faixa de nebulosidade descontínua, pois é composta de aglomerados distintos de nuvens, os quais são separados por regiões de céu claro (Mendonça e Danni-Oliveira, 2007; Uvo, 1989). A intensidade da ZCIT é variável tanto no tempo como no espaço, as precipitações excedem-se bastante, devido à umidade fornecida pela evaporação das superfícies oceânicas abaixo dela e pela evapotranspiração em algumas regiões continentais. Estudos feitos por Uvo (1989), Waliser e Gautier (1993) mostram que a posição e a intensidade da ZCIT estão intimamente relacionadas às condições de superfície do oceano e da atmosfera.

Elevadas quantidades pluviométricas, uma vez atingidas, geram grandes transtornos para a agricultura e pecuária, inclusive, grandes centros urbanos que não estão preparados economicamente e estruturalmente para receber estes volumes d'água. Neste contexto, a intensidade das chuvas ou até mesmo sua escassez, se torna mais um grande problema que a população mundial tem que enfrentar em meio a um cenário de enchentes, pessoas desabrigadas e muitas mortes. Desta forma, a ZCIT representa o sistema atmosférico com um potencial de causar grandes quantidades de precipitação sobre as áreas continentais tropicais, inclusive, sobre as grandes metrópoles, onde a população é mais acentuada. Dentre as maiores cidades localizadas na região de atuação da ZCIT, destacam-se Belém, no Brasil; Nairóbi, no Quênia; e Jakarta, na Indonésia que serão foco de estudo deste trabalho. Assim, este estudo se propõe a investigar a variabilidade da precipitação em tempo-escala associada à ZCIT no hemisfério sul, bem como a sua interação com fenômenos atmosféricos de múltiplas escalas de tempo.

\section{DADOS E METODOLOGIA}

Neste tópico serão apresentados os seguintes itens: a área de estudo, o material utilizado e as ferramentas matemáticas e estatísticas usadas para a análise dos dados observacionais de precipitação para o período de 1999 a 2008.

\section{1. Área de estudo}

A área de estudo foi selecionada por representar espacialmente a posição da variação espacial da ZCIT relacionada à sua atuação em grandes metrópoles, com semelhantes faixas de latitude e em diferentes continentes na faixa tropical. A Figura 1 mostra a localização das cidades de Belém na América do Sul (lat = $1^{\circ} 28^{\prime} 22^{\prime \prime} \mathrm{S}$, lon $=48^{\circ} 30^{\prime} 53^{\prime \prime}$ W), Nairóbi no leste da África (lat $=1^{\circ} 16^{\prime} 00^{\prime \prime} \mathrm{S}$, lon $=36^{\circ} 49^{\prime}$ $00^{\prime \prime}$ E) e Jakarta no sul da Ásia (lat $=6^{\circ} 12^{\prime} 97^{\prime}$ ' S, lon $=106^{\circ}$ $50^{\prime} 27^{\prime \prime}$ E). Estes locais apresentam diferentes composições de superfície combinadas à atuação da ZCIT, durante o solstício de verão e equinócio de outono.

\subsection{Dados}

Neste trabalho foram utilizados dados diários de precipitação (PRP) que posteriormente foram transformados em dados mensais para Belém (lat = $1^{\circ} 28^{\prime} 22^{\prime \prime} \mathrm{S}$, lon $=48^{\circ} 30^{\prime} 53^{\prime \prime} \mathrm{W}$ ), Jakarta (lat $=6^{\circ} 12^{\prime} 97^{\prime \prime} \mathrm{S}$, lon $=106^{\circ} 50^{\prime} 27^{\prime \prime}$ E) e Nairóbi (lat $=1^{\circ}$ $16^{\prime} 00^{\prime \prime} \mathrm{S}$, lon=36 49'00” E), durante o período de 1999 a 2008. Os dados meteorológicos internacionais foram fornecidos pelo Centro de Previsão de Tempo e Estudos Climáticos do Instituto Nacional de Pesquisas Espaciais (CPTEC/INPE) e os dados nacionais pelo Instituto Nacional de Meteorologia (INMET). Os dados de precipitação para a cidade de Jakarta apresentaram valores apenas para o mês de janeiro (2000), logo a ausência de dados ocorre no período de fevereiro a dezembro de 2000, devido falhas na continuidade dos dados. Além desses, foram utilizados os dados mensais de radiação de onda longa (ROL) para o período de 1999 a 2008, disponibilizados pelo National Centers for Environmental Prediction/National Center for Atmospheric Research (NCEP/NCAR), a fim de se estudar a variabilidade mensal da atividade convectiva. Estes dados foram obtidos da média diária de passagens diurna e noturna do satélite de órbita polar da NOAA e representa a atividade convectiva, com valores menores do que $240 \mathrm{~W} / \mathrm{m}^{2}$ (Liebmann e Smith, 1996).

\subsection{Método}

A partir da precipitação mensal foram feitos os cálculos da precipitação em percentagem (\%) nas escalas de tempo anual e sazonal, com o intuito de analisar a representação do 


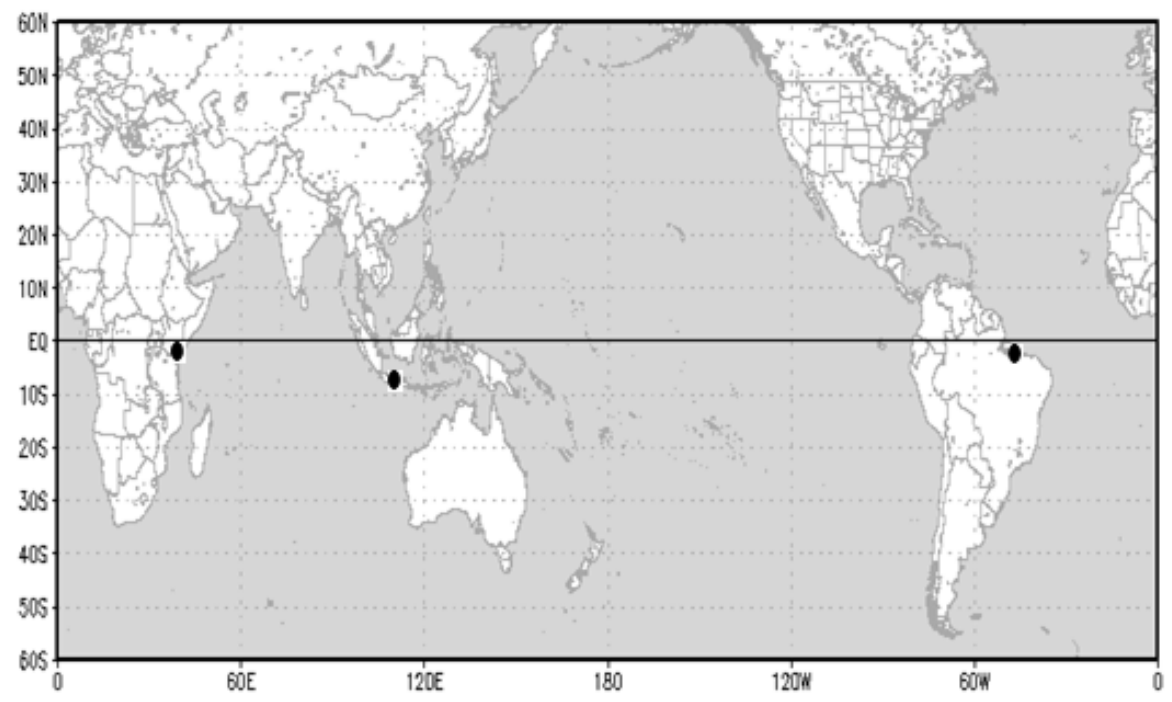

Figura 1 - Localização das cidades estudadas (pontos pretos), Nairóbi no leste da África; Jakarta no extremo sul da Ásia; Belém na América do Sul.

quantitativo da precipitação. Para a escala anual (sazonal), cada ano foi obtido da seguinte forma: utilizou-se o acumulado anual (sazonal) de precipitação de todos os anos dividido pelo acumulado anual (sazonal) de cada ano específico, assim foi encontrado o total anual (sazonal) em percentagem (T.A \%). Como Jakarta apresenta uma falha nos dados no ano de 2000, a obtenção dos percentuais médios, anual e sazonal, levou em consideração o total de 9 anos da série, e não de 10 anos como nas outras duas cidades.

No caso da escala sazonal foram estabelecidos dois períodos distintos para cada ano, um chuvoso de Janeiro a Maio e outro menos chuvoso de Junho a Dezembro (Uvo, 1989; Waliser e Gautier, 1993). O período chuvoso visa representar a atuação da ZCIT, durante o verão e outono austral. Apesar de este ser considerado por muitos autores o principal sistema precipitante de grande escala que atua na Amazônia (Leal e De Souza, 2011; Uvo, 1989), outros sistemas de meso escala e de escala sinótica também influenciam de forma significativa nas chuvas da região como Linhas de Instabilidade (LI), Zona de Convergência do Atlântico Sul (ZCAS) e Vórtices Ciclônicos de Altos Níveis (VCAN), (Cohen et al., 1995; Ramirez, 1996; Machado et al., 2000).

Os episódios de ENOS observados no período de 1999 a 2008 foram utilizados para a análise da variabilidade da precipitação anual, sazonal e mensal, visto que as áreas em estudos sofrem influência da circulação atmosférica zonal de Walker, conforme mencionado na Tabela 1.

Além disso, utilizou-se o método matemático da Transformada em Ondeletas (TO) para detectar as oscilações atmosféricas de múltiplas escalas de tempo associadas à precipitação e a ROL mensal em Belém, Nairóbi e Jakarta para o período de 1999 a 2008. Apesar das séries temporais
Tabela 1 - Episódios de ENOS para o período de 1999 a 2008.

\begin{tabular}{c|c|c|c}
\hline Ano & Meses & Fase & Intensidade \\
\hline $98 / 99$ & Dez-Jan & Neutro & - \\
\hline $99 / 00$ & Jan-Dez & LN & Fraca \\
\hline $00 / 01$ & Out-Fev & LN & Fraca \\
\hline $01 / 02$ & Mar-Abr & Neutro & - \\
\hline $02 / 03$ & Mai-fev & EM & Moderado \\
\hline $03 / 04$ & Abr-Mai & Neutro & - \\
\hline $04 / 05$ & Jun-Fev & Neutro & - \\
\hline $05 / 06$ & Mar-Jul & Neutro & - \\
\hline $06 / 07$ & Ago-Jan & EM & Fraco \\
\hline $07 / 08$ & Set-Mai & LN & Moderado \\
\hline
\end{tabular}

Fonte: (http://www.cpc.ncep.noaa.gov/products/precip/CWlink/MJO/ enso.shtml)

utilizadas representarem de algum modo a precipitação mensal, a ROL apresenta variável temporal mais adequada para a análise de múltiplas escalas, devido à sua estrutura temporal apresentar simetria e assimetria dos valores diferentes de zero, isto se assemelha ao comportamento da função ondeleta-mãe Morlet que faz a decomposição por escala do sinal estudado. No caso da PRP mensal para Nairóbi e Jakarta observam-se valores consecutivos iguais a zero, que podem causar alguma limitação na análise tempo-escala. Por isso, utilizou-se a ROL e a PRP para juntamente investigar os processos de multiescala relacionados com a precipitação da ZCIT em diferentes pontos do globo. Além disso, a ROL representa a estimativa de atividade convectiva, sendo utilizada neste estudo para contribuir no 
entendimento das múltiplas escalas associada a precipitação observada (Liebmann e Smith, 1996).

A análise da TO permitirá o entendimento da composição do sinal mensal da precipitação de cada localidade, juntamente com os mecanismos atmosféricos de diferentes escalas de tempo e suas interações, responsáveis pela modulação da chuva local.

A Transformada em Ondeletas (TO) é uma técnica de análise do sinal em tempo-escala usada para análise de sinais não-estacionários, desenvolvida a partir do final dos anos 80 , como uma forma de projetar em escalas, funções temporais (Daubechies, 1990). Este método é baseado na teoria de grupos e nas funções quadraticamente integráveis (energia finita), permitindo decompor um sinal dependente do tempo em ambos, tempo e escala. A TO da Função $f(t) \in L^{2}(\mathfrak{R})$ é definida pela Expressão (Farge, 1992):

$$
W_{\psi} f(a, b)=\frac{1}{\sqrt{a}} \int_{-\infty}^{+\infty} f(t) \psi\left(\frac{t-b}{a}\right) d t
$$

onde $a \neq 0 \in \mathfrak{R}^{+}$e $b \in \mathfrak{R}$.

A TO foi utilizada em vários estudos, desde a escala climática, El Niño Oscilação Sul a escalas micrometeorológicas, com análise de processos turbulentos (Gu e Philander, 1995; Weng e Lau, 1994). Para este estudo, a Função principal, ou seja, a função ondeleta-mãe utilizada é a Morlet, que por ser complexa fornece informações a respeito da energia (amplitude) e fase (parte real), segundo Weng e Lau (1994). Esta Função possui a seguinte forma:

$$
g(t)=e^{i \omega_{0} t} e^{-t^{2} / 2}
$$

A função ondeleta-mãe foi escolhida por ser a mais adequada à captação de sinais atmosféricos com características simétricas e assimétricas de maneira contínua ao longo do tempo (Daubechies, 1992; weng e Lau, 1994; Torrence e Compo, 1998). Desta forma, neste estudo foi analisada a fase da ondeleta da precipitação mensal, ou seja, a parte real da função Morlet. O cálculo dos coeficientes de ondeleta foi obtido através da execução de um algorítmo e visualizado através de softwares.

Para a análise dos escalogramas de fase de ondeleta (tempo versus escalas) de precipitação e de radiação de onda longa foram adicionados dados no início e no final da série para que os efeitos de borda causados pelo cone de influência da TO sejam suavizados, conforme sugerido por Torrence e Compo (1998). Assim, pode-se analisar a partir da escala de 2-3 meses que é intrassazonal (menor), da escala de 12 meses, que é a anual (intermediária), e da escala de 40-70 meses, que se denomina de interdecadal (maior).

\section{RESULTADOS E DISCUSSÕES}

\subsection{Percentual de precipitação}

A partir dos totais pluviométricos mensais e anuais, foram calculados os percentuais de precipitação para cada cidade. De acordo com a Tabela 2, verifica-se que Belém apresenta uma regularidade no percentual de precipitação entre $8 \%$ e $12 \%$, com uma média de $10 \%$ nos dez anos estudados. O período de janeiro a maio apresenta uma média de 10,2 \% de precipitação em todos os anos da série, e o de junho a dezembro, uma média de 10\%. Em 2006 ocorreu maior percentual de precipitação (12\%). Apesar deste ano não ter sofrido a influência do fenômeno ENOS, alguns estudos mostram que a ZCIT pode interagir com fenômenos meteorológicos de meso escala, como as linhas de instabilidade (LI), que são responsáveis por, aproximadamente, $45 \%$ da precipitação do leste paraense, com máxima frequência entre os meses de abril e agosto (Cohen, 1989; Cohen et al., 1995).

As características locais de cada lugar são de grande relevância para a determinação de um regime chuvoso de uma localidade. Belém está envolvida por uma região com grande potencial hídrico, devido à grande abundância de rios e florestas e a proximidade com o oceano. Segundo Mota e

Tabela 2 - Percentual de precipitação para o período de 1999 a 2008. Onde: T.A. - Total Anual; JA - Janeiro/JU - Junho/MA - Maio/DE - Dezembro

\begin{tabular}{c|c|c|c|c|c|c|c|c|c}
\cline { 2 - 10 } & \multicolumn{3}{c|}{ BELÉM } & \multicolumn{3}{c|}{ JAKARTA } & \multicolumn{3}{c}{ NAIRÓBI } \\
\cline { 2 - 11 } & T. A.(\%) & $\begin{array}{c}\text { JA a MA } \\
(\%)\end{array}$ & $\begin{array}{c}\text { JU a DE } \\
(\%)\end{array}$ & T. A.(\%) & $\begin{array}{c}\text { JA a MA } \\
(\%)\end{array}$ & $\begin{array}{c}\text { JU a DE } \\
(\%)\end{array}$ & T. A.(\%) & $\begin{array}{c}\text { JA a MA } \\
(\%)\end{array}$ & $\begin{array}{c}\text { JU a DE } \\
(\%)\end{array}$ \\
\hline $\mathbf{1 9 9 9}$ & 9 & 64 & 36 & 10 & 48 & 52 & 11 & 19 & 81 \\
\hline $\mathbf{2 0 0 0}$ & 11 & 64 & 36 & \multicolumn{2}{c|}{ AUSÊNCIA DE DADOS } & 10 & 75 & 25 \\
\hline $\mathbf{2 0 0 1}$ & 10 & 60 & 40 & 4 & 74 & 26 & 14 & 78 & 22 \\
\hline $\mathbf{2 0 0 2}$ & 9 & 59 & 41 & 8 & 44 & 56 & 8 & 43 & 57 \\
\hline $\mathbf{2 0 0 3}$ & 9 & 58 & 42 & 14 & 65 & 35 & 7 & 61 & 39 \\
\hline $\mathbf{2 0 0 4}$ & 9 & 64 & 36 & 8 & 88 & 12 & 13 & 41 & 59 \\
\hline $\mathbf{2 0 0 5}$ & 11 & 59 & 41 & 5 & 82 & 18 & 5 & 79 & 21 \\
\hline $\mathbf{2 0 0 6}$ & 12 & 59 & 42 & 10 & 63 & 37 & 16 & 56 & 44 \\
\hline $\mathbf{2 0 0 7}$ & 10 & 58 & 42 & 21 & 64 & 36 & 10 & 40 & 60 \\
\hline $\mathbf{2 0 0 8}$ & 11 & 67 & 33 & 20 & 87 & 13 & 6 & 71 & 29 \\
\hline MÉDIA & 10 & 61 & 39 & 12 & 71 & 29 & 10 & 56 & 44 \\
\hline
\end{tabular}


Mendonça (2004), esses fatores locais contribuem para elevar a atividade convectiva, havendo assim uma melhor distribuição da precipitação em todos os anos. Isso explica porque Belém apresenta essa pouca variação anual e estações chuvosas mais acentuadas do que os períodos menos chuvosos, com relação à Jakarta e Nairóbi.

Jakarta apresenta uma média de precipitação anual de $12,2 \%$ e uma média sazonal, de janeiro a maio, de $11,2 \%$. Isto se observa devido a concentração das chuvas ocorrerem durante os meses de verão e outono, elevando assim a representação da média sazonal. Em relação ao período de junho a dezembro, a precipitação representa 11,3 \% do total anual. 2006, 2007 e 2008 foram os anos com tiveram o maior percentual de precipitação. As numerosas ilhas que envolvem Jakarta apresentam características físicas e geográficas que favorecem a convecção nessa região da Indonésia, devido à pequena área oceânica que sofre um estreitamento por essas pequenas porções continentais gerando aquecimento diferencial. Alguns estudos mostram que o regime de precipitação da Ilha de Java e adjacências é caracterizado, principalmente, pelas monções (Ramage, 1971), pelo ciclo do fenômeno ENOS (Hendon e Liebmann, 2003; Philander, 1989; Ropelewski e Halpert, 1992) e pela Oscilação Intrassazonal Madden-Julian (Madden e Julian, 1971,1972, 1994). A Indonésia apresenta duas fases de monção a cada ano, a fase chuvosa de dezembro a abril, que coincide com a presença da ZCIT nesta região (Asnani, 1993) e a fase seca de maio a novembro.

No caso de Nairóbi, a média anual da série de dados é igual à de Belém $(10 \%)$, porém a média do primeiro período de atuação da ZCIT (janeiro a maio) é de $10,1 \%$ e do período fora de atuação do fenômeno (junho a dezembro) é de $10 \%$. A precipitação associada à ZCIT no leste do continente africano apresenta uma dinâmica bastante complexa em função de fatores locais como a topografia, a heterogeneidade de vegetação (deserto, savana e floresta), lagos e a proximidade com o oceano Índico. Segundo Nicholson (1993), na capital do Quênia, Nairóbi, as chuvas mais intensas ocorrem durante os meses de março-maio e chuvas de curta duração de outubro-novembro. Contudo, esta última está relacionada com a variabilidade interanual. Outro mecanismo responsável por essa distribuição de precipitação pode estar associada à Temperatura da Superfície do Mar (TSM) do Atlântico Tropical e do Oceano Índico, que por sua vez estão fracamente acoplados ao ENOS. Ainda segundo Nicholson (1993), para a região como um todo, o Oceano Atlântico parece afetar mais as chuvas do que o Oceano Índico.

Os anos de 2007 e 2008 tiveram episódios de La Niña até o mês de junho (CPC/NOAA), logo um ramo ascendente da célula de Walker se posiciona próximo as ilhas da Indonésia, e o outro ramo ascendente sobre a Amazônia, o que favorece a convecção nessas duas regiões da faixa equatorial. No entanto, na África a circulação de Walker é modificada, diminuindo as chuvas nesta área.

A Figura 2 mostra que as três cidades estudadas apresentam uma variabilidade anual de precipitação bem acentuada. Belém mostra totais pluviométricos acima de 3.000 $\mathrm{mm}$ em seis dos dez anos estudados, com o menor valor de $2.600 \mathrm{~mm}$ em 1999. Nairóbi apresenta os menores valores de precipitação, com um máximo em torno de $2.600 \mathrm{~mm}$ (2006) e mínimo de $800 \mathrm{~mm}$ (2005). Por outro lado, Jakarta mostra maiores variações de amplitude do que as demais cidades. As maiores precipitações ocorrem em 2007 (4259,4 mm) e 2008 (3962,5 mm), e a menor em 2000, porém como este ano apresenta falhas nos dados analisados, o ano com menor valor de PRP foi 2001 (877,6 mm).

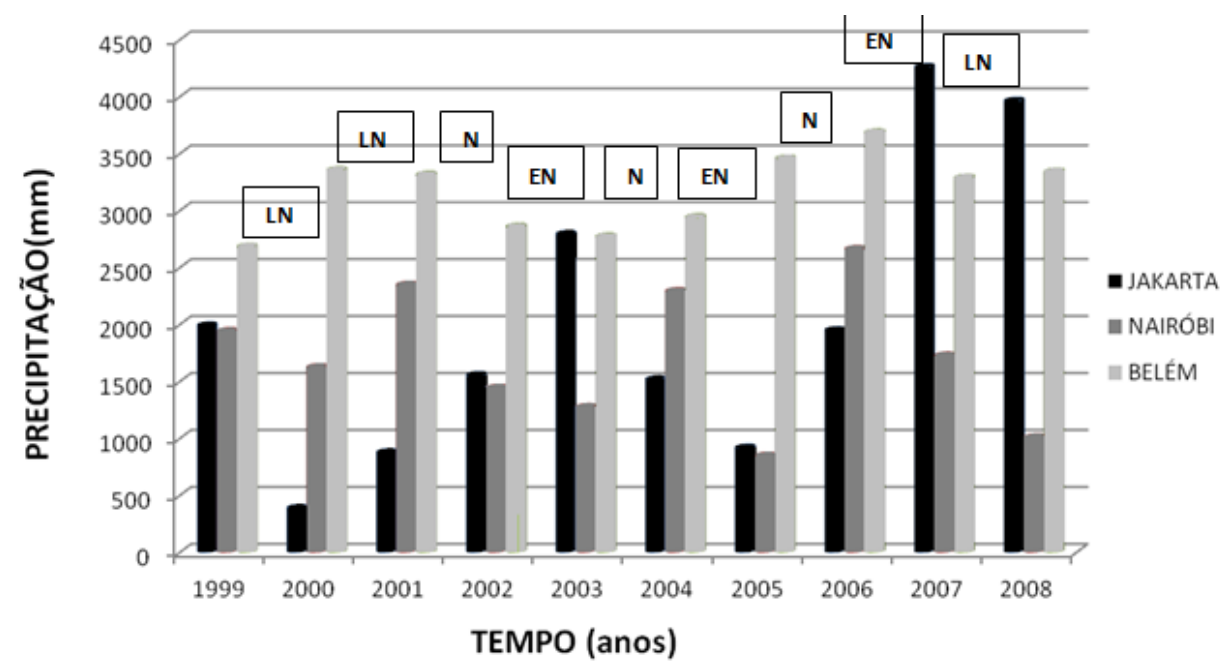

Figura 2 - Variação tempo-espaço da precipitação anual acumulada para Belém, Jakarta e Nairóbi, durante o período de 1999 a 2008 . Onde EN: El Niño; LN: La Niña; N: normal, para as transições de cada ano nesta década. 
Jakarta apresenta o segundo maior volume pluviométrico, sendo que ultrapassa Belém em três anos, 2003, 2007 e 2008, com valores máximos de até $4.000 \mathrm{~mm}$. No entanto, esta cidade apresenta valores muito variados de precipitação ao longo de 10 anos. Nairóbi, apesar da pouca precipitação acumulada em comparação com as outras duas cidades, apresenta pouca variação com valores acima de $1000 \mathrm{~mm}$ em $80 \%$ dos dados obtidos. Belém é a cidade que apresenta menor variação ao longo do período, portanto, tem maior frequência e volume de chuvas, em relação às outras três cidades estudadas.

\subsection{Análise de multiescala da precipitação mensal}

Esta análise de multiescala da precipitação mensal para Belém, Nairóbi e Jakarta visa detectar as oscilações atmosféricas responsáveis pelo comportamento da chuva no período de 1999 a 2008. Assim, as Figuras 3, 4 e 5 apresentam as séries temporais de precipitação e os escalogramas de fase da ondeleta de precipitação e radiação de onda longa.

\subsubsection{Belém}

As Figuras 3a e 3b são concordantes do ponto de vista das fases das escalas intrassazonal, anual e interdecadal relacionadas às oscilações de ROL e de PRP para Belém-PA. A escala anual mostra-se mais energética com relação às outras, porém os anos de 2001, 2002, 2006, 2007 e 2008 apresentam oscilações de ROL e PRP em torno de 2-3 meses, mais evidente na figura $3 \mathrm{~b}$, o que pode caracterizar a atuação da escala intrasazonal para estes períodos.

Nota-se ainda que os eventos de El Niño-Oscilação Sul (ENOS), com El Niño (EN) com intensidade moderada em 2002-2003 e em 2007- 2008 a La Niña moderada (LN) (ver tabela 1) estiveram associados com as fases de redução e aumento de precipitação na escala anual. As oscilações sazonais e intrasazonais aparecem com maior intensidade no período chuvoso, durante os anos de 2000-2001, 2001-2002, 20052006, 2006-2007 e 2007-2008. Estas oscilações podem está relacionadas com a variabilidade interanual dos anos normais e de La Niña. Segundo Lau e Chan (1988) em anos extremos de El Niño as oscilações de Madden e Julian desaparecem em diferentes regiões tropicais. No entanto, segundo Oliveira e Vitorino (2011), durante os anos normais e de LN as oscilações intrasazonais são freqüentes sobre de Belém-PA.

\subsubsection{Nairóbi}

O escalograma de fase de ROL para Nairóbi (Figura 4a) mostra que as oscilações de maior magnitude encontram-se na escala anual, com oscilações semianuais aperiódicas, e interdecadal (40 meses). No caso do escalograma de PRP observa-se a atuação das escalas interdecadal, com maior periodicidade, e, anual, interanual, semianual e intrassazonal com grande variação temporal, principalmente em anos de ocorrência de ENOS, com fraca intensidade, como por exemplo, os anos de 1999-2000, 2000-2001 e 2006-2007 (Figura 4 b). Observacionalmente, a maioria das oscilações de múltiplas escalas são diferentes e irregulares entre si, para ROL e PRP. No entanto, as escalas relacionadas as oscilações interdecadais são evidentes tanto na ROL como na PRP. A divergência dos escalogramas da ROL e da PRP surgem para a observação das oscilações de frequência mais alta, como anual, semianual e a intrassazonal, onde a PRP mostra maior variabilidade temporal. Esta por sua vez indica que a PRP expressa as condições da superfície na região de Nairóbi. Isto de certa forma concorda com Nicholson (1993), quando ressalta que a cobertura do solo e as características físicas geográficas podem ser o diferencial no processo convectivo local.

(a)

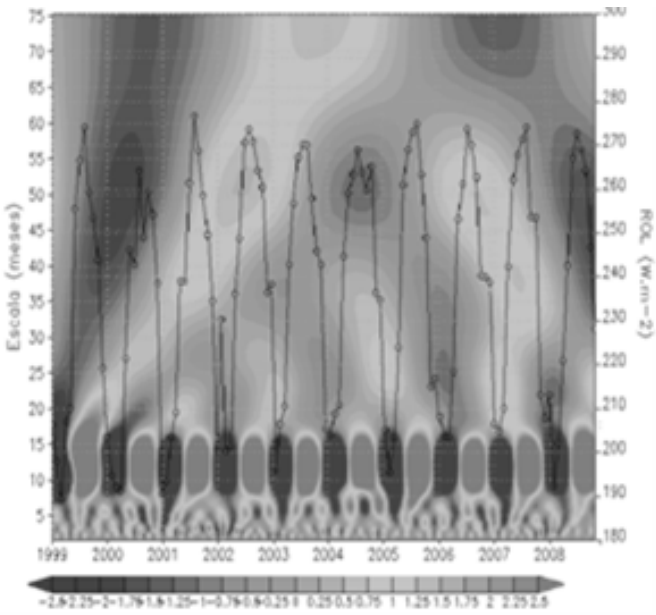

(b)

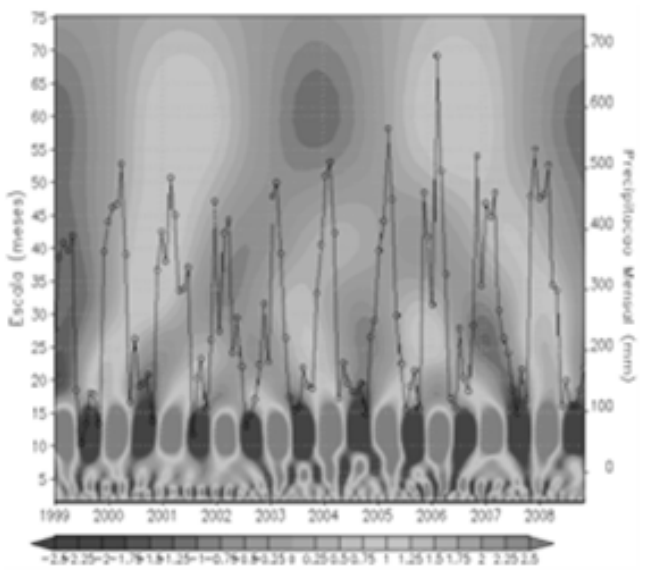

Figura 3 - Escalograma de fase da ondeleta Morlet e a série temporal observada para Belém, durante 1999 a 2008, para a (a) fase da ROL (sombreado) e série da ROL (linha), e para a (b) fase da PRP (sombreado) e série da PRP (linha), respectivamente (sombreado: tons escuros fase negativa e tons claros fase positiva). 
(a)

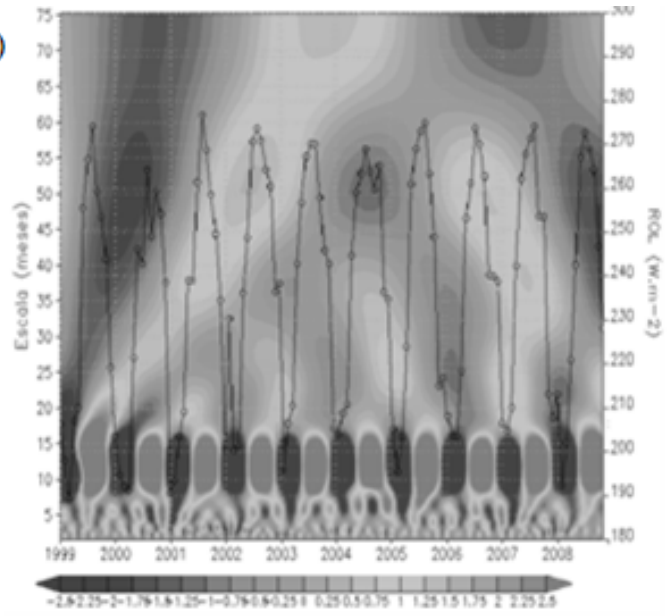

(b)

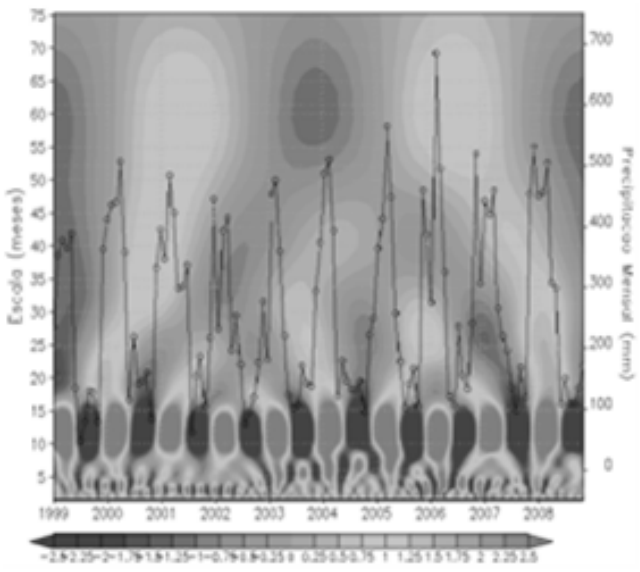

Figura 4 - Escalograma de fase da ondeleta Morlet e a série temporal observada para Nairóbi, durante 1999 a 2008, para a (a) fase da ROL (sombreado) e série da ROL (linha), e para a (b) fase da PRP (sombreado) e série da PRP (linha), respectivamente (sombreado: tons escuros fase negativa e tons claros fase positiva).

\subsubsection{Jakarta}

Observa-se na série temporal de ROL e de precipitação mensal observada para Jakarta (Figuras 5 a e 5 b) que existe concordância entre as variáveis no maior número de eventos de chuva, exceto para 2002-2003 e 2006-2007. Isto pode estar relacionado com a ocorrência dos episódios de EN (LN) (CPC/NCEP/NOAA), que segundo Aldrian et al. (2007) reduz (aumenta) a precipitação na região da Indonésia. Com relação a PRP, a ROL apresenta menor amplitude das múltiplas escalas observadas no escalograma de fase, isto pode estar relacionado com a subestimativa na ROL. Desta forma, esta apresenta pouca evidência das oscilações associadas às escalas interdecadal, interanual e intrazonal. Segundo Silva Dias et al. (2002) e Liebmann e Smith (1996), a ROL p ode subestimar e superestimar a atividade convectiva na região tropical. No (a)

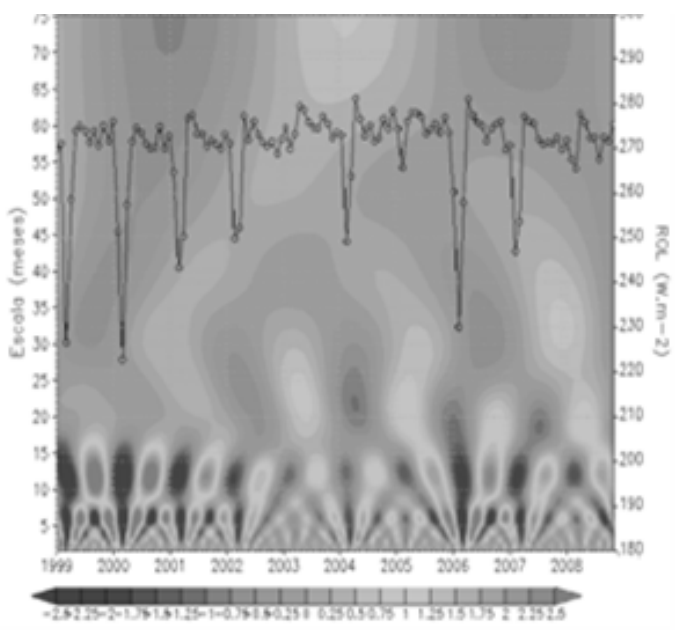

(b)

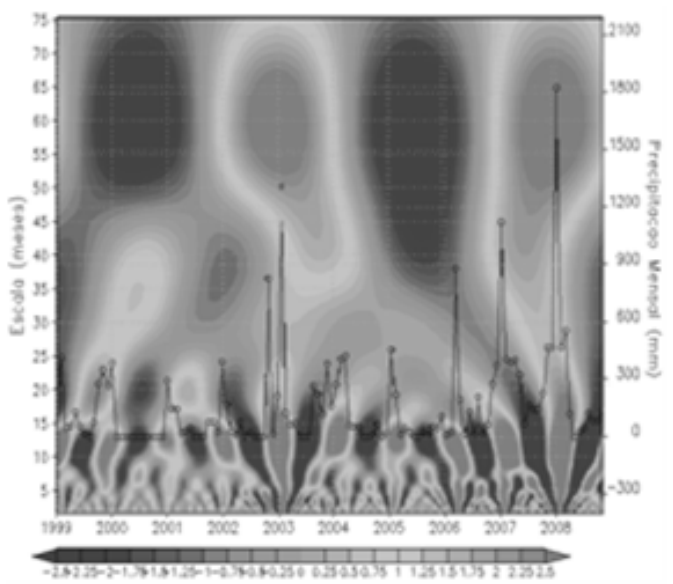

Figura 5 - Escalograma de fase da ondeleta Morlet e a série temporal observada para Jakarta, durante 1999 a 2008, para a (a) fase da ROL (sombreado) e série da ROL (linha), e para a (b) fase da PRP (sombreado) e série da PRP (linha), respectivamente (sombreado: tons escuros fase negativa e tons claros fase positiva).

entanto, em geral, a mesma se apresenta como um bom indicador de convecção tropical.

Apesar de Jakarta apresentar um quantitativo de precipitação maior que as demais cidades, esta mostra irregularidade temporal na precipitação (Figura 5 b). Um aspecto que pode ser observado é que os extremos mensais estão relacionados com os episódios de ENOS, como por exemplo, na LN de 2007-2008, com PRP de 1800 mm/mês e no EN de 20022003 alguns meses com ausência de chuva. É importante lembrar que em 2000 o zero de precipitação se deve a falta de dados no período. Diferente de Nairóbi, Jakarta apresenta um ciclo anual (12 meses) bem definido no período de 1999 a 2008 . No entanto, as oscilações de precipitação nas escalas interdecadais e intrassazonais aparecem semelhantes às de Belém.

Em geral, observou-se que as multiescalas relacionadas às oscilações de PRP e da ROL ocorrem nas escalas interdecadal, 
interanual e intrassazonal, de modo diferenciado nas três cidades de estudo. No entanto, Belém e Jakarta apresentam mecanismos moduladores de chuva associados a semelhantes escalas de tempo, devido às condições de superfície das regiões que envolvem ambas as cidades serem muito parecidas, como a proximidade de florestas e de oceanos adjacentes. Por outro lado, a geografia e as condições de superfície de Nairóbi podem ser as características marcantes para os extremos de precipitação e sua irregularidade.

Contudo, a qualidade da estação chuvosa relacionada à ZCIT, fenômeno precipitante tropical de escala global que atua em todos os continentes, depende fortemente da modulação das oscilações interdecadais e das condições locais de superfície da cada região. Logo, os impactos na precipitação serão bastante diferenciados dependendo do continente do globo terrestre em que este fenômeno atua.

\section{CONCLUSÕES}

O objetivo principal deste trabalho foi investigar e analisar a variabilidade tempo-espaço da precipitação observada e da radiação de onda longa relacionadas com a atuação da Zona de Convergência Intertropical em Belém, Nairóbi e Jakarta, e seus processos convectivos de múltiplas escalas de tempo. Para isso foram utilizados dados observacionais de PRP e de ROL para o período de 1999 a 2008. Com base nisso, foram obtidas as seguintes conclusões:

Em termos gerais, a ZCIT não atua da mesma forma nas três cidades estudadas, apesar de estas estarem localizadas em latitudes semelhantes e sob a atuação do mesmo sistema precipitante de grande escala;

De forma comum para as três cidades estudadas, a escala interdecadal modula a variabilidade da precipitação;

O quantitativo da precipitação na composição da variação anual, sazonal e intrassazonal evidenciam a influência do ENOS;

- Belém apresenta maior regularidade na quantidade da chuva anual e sazonal, com relação à Jakarta e Nairóbi, no entanto, para ROL, Belém e Nairóbi apresentam maior intensidade nas oscilações em torno de 12 meses (anual);

- As oscilações de múltiplas escalas de precipitação em Belém e Jakarta apresentam semelhantes escalas relacionadas aos mecanismos moduladores da chuva local, inclusive o ciclo anual marcado, durante todo o período, logo as oscilações de precipitação nas escalas em torno de 60 (5 anos), 3 a 2 meses, em Jakarta, aparecem semelhantes a Belém;

- Nairóbi, apesar de localizada em latitude análoga a Belém, não apresenta ciclo anual de modo contínuo.
No entanto, as oscilações de escala anual e interanual parecem determinar a precipitação local;

- Concordância com estudos anteriores, com relação à presença da oscilação intrassazonal (Madden e Julian) em anos de La Niña e ausência da mesma em anos de El Niño.

\section{AGRADECIMENTOS}

Os autores agradecem ao Instituto Nacional de Meteorologia (INMET), juntamente com a meteorologista Aylci Nazaré pelo fornecimento de 10 anos de dados para a cidade de Belém.

Ao Centro de Previsão de Tempo e Estudos Climáticos (CPTEC), em conjunto com o Instituto Nacional de Pesquisas Espaciais (INPE), pela atenção e generosidade de fornecer os dados meteorológicos internacionais de Nairóbi no Quênia e Jakarta na Indonésia. Além desses, agradecemos ao Laboratório de Meteorologia Sinótica da Universidade federal do Pará (UFPA) pela infraestrutura fornecida para a realização deste trabalho.

\section{REFERÊNCIAS BIBLIOGRÁFICAS}

ALDRIAN, E., DUMENIL GATES E F. H. WIDODO. Seasonal variability of Indonesian rainfall in ECHAM4 simulations and in the reanalyses: The role of ENSO. Theoretical and Applied Climatology. 87, 41-59, 2007.

ASNANI, G.C. Tropical Meteorology, Noble Printers PVT. Ltd. PUNE, India, 2v, 1993.

COHEN, J. C. P; SILVA DIAS, M. A F.; NOBRE C. A. Aspectos climatológicos das linhas de instabilidade na Amazônia. Climanálise, São José dos Campos, v. 4, n.11, p. 34-40, Nov. 1989.

COHEN, J. C. P; SILVA DIAS, M. A F.; NOBRE C. A. Environmetal conditions associated with Amazonian squall lines: a case study. Monthy Weather Review, Boston, v. 123, n.11, p. 3163-3174, Nov. 1995.

DAUBECHIES, I. Ten lectures on wavelets, SIAM, Philadelphia. 357 p. 1990

FARGE, M. The Wavelet Transform and its Applications to Turbulence. Annual Review of Fluid Mechanics, 24, 395-457. 1992

GU, D.; PHILANDER, S.G.H. Secular Changes of Annual and Interannual Variability in the Tropics during the Past Century. Journal of Climate, 8, 4: 864-876, April. 1995.

HENDON, H.; LIEBMANN, B. A composite study of the onset of the Australian summer monsoon. Journal Atmospheric Sciences, v.47, p.2227-2240, 2003.

LAU, K.M.; CHAN, P.H.: Intraseasonal and Interannual variability of tropical convection: A possible link between 
the 40-50 day oscillation and ENSO? Journal Atmospheric Sciences, 45, 506-521, 1988.

LEAL, S. V.; DE SOUZA, E. B. Desastres Naturais sobre a Amazônia e Nordeste Brasileiro associados às enchentes e inundações: O Caso de 2009. IV ENCONTRO SULBRASILEIRO DE METEOROLOGIA. Pelotas-RS, 2011.

LIEBMANN, B.; SMITH, C. A.: Description of a Complete (interpolated) Outgoing Long wave Radiation Dataset. Bulletin of the American Meteorological Society, 77, 1275-1277, 1996.

MACHADO, L. A. T.; DUVEL, J. PH; LAURENT, H.; SIQUEIRA, J. R. Meridional propagation of the convection in South America. CONGRESSO INTERNACIONAL DE MEteorologiA, 6, 2000, Chile. Anais, 2000.

MADDEN R.A.; JULIAN, P.R. Detection of a 40-50 day oscillation in the zonal wind in the tropical pacific, Journal Atmospheric Sciences, 29, 1109-1123. 1971.

MADDEN R.A.; JULIAN, P.R. Description of global-scale circulation cells in the tropics with a 40-50 day period. Journal Atmospheric Sciences, 29, 1109-1123. 1972.

MADDEN R.A.; JULIAN, P.R. Observations of the 40-50 day tropical oscillation Montlhy Weather Review, 122, 814-837, 1994.

MENDONCA. F.; DANNI-OLIVEIRA I. M. Climatologia noções básicas e climas do Brasil. São Paulo: Oficina de Textos, 2007.

MOTA, M. A. S.; MENDONÇA, M. A. Avaliação do esquema de convecção RAS utilizando o modelo CGM do CPTEC. In: CONFERÊNCIA CIENTÍFICA do LBA, 3., 2004, Brasília, DF. Anais... Brasília, DF: [s.n.], 2004.

MOURA, A.D.; SHUKLA, J. On the dynamic of droughts in Northeast Brazil: Observations theory and numerical experiments with a general circulation model. Journal Atmospheric Sciences, 38, 2653-2675, 1981.

NICHOLSON, S.E. A Review of Climate Dynamics and Climate Variability in Eastern Africa, Departament of Meteorology, Florida state University, Tallahassee, Florida, United States. 1993

OLIVEIRA, J. V.; VITORINO, M. I. Climatology Analysis El Niño - Southern Oscilation and Madden-Julian Oscilation: A 30 years Analysis (1980-2009). IV SIMPÓSIO INTERNACIONAL DE CLIMATOLOGIA, João PessoaPB, Brasil, Anais, 2011.

PHILANDER, S.G. El Niño, La Niña, and the Southern Oscillation. Academic press, INC. San Diego, California, 1989.

RAMAGE, C. S. Monsoon Meteorology. New York: Academic Press. 296p. 1971

RAMIRES, M. C. V.: Padrões climáticos dos vortices ciclônicos de altos níveis no nordeste do Brasil.
Dissertação (Mestrado em Meteorologia). Instituto Nacional de Pesquisas Espaciais, São José dos Campos, 1996.

RASMUSSON, E.M.; ARKIN, P.A. Inmteranual climate variability associated with the El Niño/ Southerm Oscilation. Coupled Ocean-Atmosphere Models, J.C.J. Nihaud Ed., Elsevier, 289-302, 1985.

ROPELEWSKI, C. F.; HALPERT, M. Global and regional scale precipitation patterns associated with the El Niño/ Southern Oscillation. Montlhy Weather Review, v.115, p.1606-1626. 1992.

SILVA DIAS, M.A.F.; RUTLEDGE, S.; KABAT, P. ; SILVA DIAS, P.L.; NOBRE, C.A.; FISCH, G.; DOLMAN, A.J.; ZIPSER, E.; GARSTANG, M.; MANZI, A.O.; FUENTES, J.; ROCHA, H.R.; MARENGO, J. A.; PLANAFATTORI, A.; SÁ, L.; ALVALÁ, R.; ANDREAE, M.; ARTAXO, P.; GIELOW,R.; GATTI, L. Clouds and rain processes in abiosphere atmosphere interation context in the Amazon region. Jornal of Geophysical Research, Estados Unidos, v.107, n.D20, p.46. 1-46.23, 2002

SOUZA, E. B.; AMBRIZZI, T. Modulation of the intraseasonal rainfall over tropical Brazil by the Madden-Julian oscillation. International Journal of Climatology, v. 26, p. 1759-1776, 2006.

TORRENCE, C.; COMPO, G.P. A practical guide to wavelet analysis. Bull. Amer. Meteor. Soc., 79, p.61-78, 1998.

UVO, C. B. A Zona de Convergência Intertropical (ZCIT) e sua relação com a precipitação na região norte do Nordeste brasileiro. 1989. 88f. Dissertação (Mestrado em Meteorologia) - Instituto Nacional de Pesquisas Espaciais -INPE. São José dos Campos-SP, 1989.

VITORINO, M.I. Oscilações intrasazonais sobre a América do Sul e Oceanos Adjacentes Utilizando a Análise de Ondeletas. Tese (Doutorado em Meteorologia) - INPE 9822 - TDI/865, SP, 2003.

VITORINO, M.I.; SILVA DIAS, P.L.; FERREIRA, N.J. Observational study of the seasonality of the submonthly and intraseasonal signal over the tropics. Meteorology and Atmospheric Physics, 94, p.17-35. 2006.

WALISER, E.W.C.;GAUTIER, C.ASatellite-derivedClimatology of the ITCZ. Journal of Climate, 6. p.2162-2174. 1993.

WEICKMAN, K.M.; LUSSKY, G.R; KUTZ BACH, J.E. Intraseasonal (30-60 Day) Fluctations of Outgoing Long Wave Radiation and $250 \mathrm{mb}$ Strem function During Northerm Winter. Amer. Meteor. Soc., 113, p. 941-961, 1985.

WENG, H.; LAU, K.M. Wavelet, period doubling, and timefrequency localization with application to organization of convection over the tropical western Pacific. Journal Atmospheric Sciences, 51, p. 2523-2541, 1994. 